\title{
Expansion of $\boldsymbol{U}_{\mathrm{PMNS}}$ and Neutrino Mass Matrix $M_{v}$ in Terms of $\sin \theta_{13}$ for Inverted Hierarchical Case
}

\author{
Subhankar Roy, Nangkham Nimai Singh \\ Department of Physics, Gauhati University, Guwahati, India \\ Email: meetsubhankar@gmail.com,nimai03@yahoo.com
}

Received January 1, 2013; revised January 31, 2013; accepted February 8, 2013

Copyright (C) 2013 Subhankar Roy, Nangkham Nimai Singh. This is an open access article distributed under the Creative Commons Attribution License, which permits unrestricted use, distribution, and reproduction in any medium, provided the original work is properly cited.

\begin{abstract}
The recent observational data supports the deviation from Tri-bimaximal (TBM) mixing. Different theories suggest the interdependency among the observational parameters involving the mixing angles. On phenomenological ground, we try to construct the PMNS matrix, $U_{\mathrm{PMNS}}$ with certain analytic structure satisfying spontaneously the unitary condition, in terms of a single observational parameter $\sin \theta_{13}$. We hypothesise the three neutrino masses, $m_{i}$ as functions of $\sin \theta_{13}$ and then construct the neutrino mass matrix $M_{v}$ with certain exact and expandable form. We assume the convergence of the model to TBM mixing when $\theta_{13}$ is taken 0 . The mass matrix so far obtained can be employed for various applications including the estimation of matter-antimatter asymmetry of the universe.
\end{abstract}

Keywords: Inverted Hierarchy; Tri-Bimaximal Mixing; Neutrino Masses; $\mu-\tau$ Symmetry

\section{Introduction}

Recent results published by Double Chooz [1], Daya Bay [2], RENO [3], T2K [4] and MINOS [5] collaborations assure relatively large reactor angle $\left(\theta_{13}\right)$. Also recent global neutrino data analysis [6] insists on $\theta_{23}<\pi / 4$. Tri-bimaximal mixing [7] is associated with $\theta_{13}=0$, and $\theta_{23}=\pi / 4$. This symmetry has a strong theoretical support because of its relation with so called $\mu-\tau$ symmetry of neutrino mass matrix. $\mu-\tau$ symmetry, in turn is associated with $A_{4}$ [8-12], one of the candidates of discrete flavour symmetry groups. But in order to comply with the recent experimental results, some perturbations have to be introduced in this mixing pattern. Whether the corrections $[13,14]$ are needed or a new mixing scheme is to be introduced, is still an open question [15].

Literature $[16,17]$ shows the dependency of the mixing angles on one another. If this is true, then we are allowed to choose a single parameter capable of describing all the three mixing angles.

We move a step ahead and express the three masses under this parameter. This helps us to define a simplified neutrino mass model with a single parameter only.

Out of all the three observational parameters concerning the mixing angles $\sin \theta_{13}$ is the smallest one. So, we choose $\sin \theta_{13}$ as the guiding parameter. We consider tribimaximal mixing pattern and $\mu-\tau$ symmetry as the first approximation. Hence the model is supposed to produce T. B. M mixing when we put $\sin \theta_{13}=0$. We try to keep the structure of the three rotation matrices $U\left(\theta_{13}\right), U\left(\theta_{12}\right)$ and $U\left(\theta_{23}\right)$ in analytical form so that they can satisfy the unitary condition $\left[U\left(\theta_{i j}\right)\right]^{\top} U\left(\theta_{i j}\right)=I$, without any prior approximation.

We start with the following ansatz,

$$
\begin{gathered}
S_{13}=\epsilon, \\
s_{12}=\frac{1}{\sqrt{3}}-\frac{\epsilon}{5}, \\
s_{23}=\frac{1}{\sqrt{ } 2}-\frac{\epsilon}{2} .
\end{gathered}
$$

where, $s_{i j}=\sin \theta_{i j}$, and then construct the PMNS mixing matrix and then the neutrino mass matrix in the usual way.

\section{Construction of the PMNS Matrix}

We consider the charged lepton mass matrix to be diagonal. Hence we can choose $U_{\mathrm{PMNS}}=U_{v}$. We propose the three rotation matrices as: 
(4)

$$
\begin{aligned}
& u_{\tau 2}=\left(\frac{1}{\sqrt{2}}-\frac{\epsilon}{2}\right) a(\epsilon)-\frac{1}{30} \epsilon(3 \epsilon-5 \sqrt{3}) b(\epsilon) \mathrm{e}^{\mathrm{i} \delta}, \\
& u_{\tau 3}=\frac{1}{2}\left(1-\epsilon^{2}\right) b(\epsilon),
\end{aligned}
$$

and,

$$
\begin{aligned}
& a(\epsilon)=\left(\frac{1}{3}+\frac{2 \epsilon}{5 \sqrt{3}}-\frac{\epsilon^{2}}{25}\right)^{\frac{1}{2}}, \\
& b(\epsilon)=\left(2-\epsilon^{2}+2 \sqrt{2} \epsilon\right)^{\frac{1}{2}}, \\
& c(\epsilon)=\left(150+30 \sqrt{\epsilon}-9 \epsilon^{2}\right)^{\frac{1}{2}}, \\
& d(\epsilon)=\left(\frac{50}{3}+\frac{10 \epsilon}{\sqrt{3}}-\epsilon^{2}\right)^{\frac{1}{2}} .
\end{aligned}
$$

It can be checked that,

$$
\left[U\left(\theta_{i j}\right)\right]^{\dagger} U\left(\theta_{i j}\right)=\left[U_{\mathrm{PMNS}}\right]^{\dagger} U_{\mathrm{PMNS}}=\left(\begin{array}{lll}
1 & 0 & 0 \\
0 & 1 & 0 \\
0 & 0 & 1
\end{array}\right) .
$$

And we get,

$$
\begin{aligned}
& \tan ^{2} \theta_{12}=\left|\frac{u_{e 2}}{u_{e 1}}\right|^{2}=\frac{25-10 \sqrt{3} \epsilon+3 \epsilon^{2}}{50+10 \sqrt{3} \epsilon-3 \epsilon^{2}} \\
& \tan ^{2} \theta_{12}=\left|\frac{u_{e 2}}{u_{e 1}}\right|^{2}=\frac{25-10 \sqrt{3} \epsilon+3 \epsilon^{2}}{50+10 \sqrt{3} \epsilon-3 \epsilon^{2}}
\end{aligned}
$$

After interpreting the above two relations in terms of $\sin \theta_{13}$, we have,

$$
\begin{aligned}
& \tan ^{2} \theta_{12}=\frac{1}{2}-\frac{1}{2} \sin \theta_{13}+\frac{1}{4} \sin ^{2} \theta_{13}-\frac{2}{25} \sin ^{3} \theta_{13}, \\
& \tan ^{2} \theta_{23}=1-\frac{13}{5} \sin \theta_{13}+2 \sin ^{2} \theta_{13}-2 \sin ^{3} \theta_{13} .
\end{aligned}
$$

$U_{\text {PMNS }}$ for $\epsilon=0$ and $\epsilon=0.156$ are shown below,

$$
U_{\mathrm{TBM}}=\left(\begin{array}{ccc}
\sqrt{\frac{2}{3}} & -\sqrt{\frac{1}{3}} & 0 \\
\sqrt{\frac{1}{6}} & \sqrt{\frac{1}{3}} & -\sqrt{\frac{1}{2}} \\
\sqrt{\frac{1}{6}} & \sqrt{\frac{1}{3}} & \sqrt{\frac{1}{2}}
\end{array}\right),
$$

$$
U=\left(\begin{array}{ccc}
0.8274 & -0.5395 & 0.156 \mathrm{e}^{-\mathrm{i} \delta} \\
0.4245+0.0822 \mathrm{e}^{\mathrm{i} \delta} & 0.6511-0.0536 \mathrm{e}^{\mathrm{i} \delta} & -0.6214 \\
0.3435-0.1015 \mathrm{e}^{\mathrm{i} \delta} & 0.5270+0.0662 \mathrm{e}^{\mathrm{i} \delta} & 0.7678
\end{array}\right)
$$


This is clear from the above analysis that $\tan ^{2} \theta_{12}=0.5$ and $\tan ^{2} \theta_{23}=1$, if $\sin \theta_{13}=\epsilon=0$ (TBM mixing). At $\epsilon=0.155$ (N. H), 0.156 (I. H) (the best-fit value of $\sin \theta_{13}$ ) [6], we get $\tan ^{2} \theta_{12}=0.425$ and $\tan ^{2} \theta_{23}=0.657,0.654$, which are very close to the best fit results [6]: $\tan ^{2} \theta_{12}=$ $0.443\left(\mathrm{~N} . \mathrm{H}\right.$ or I. H) and $\tan ^{2} \theta_{23}=0.628(\mathrm{~N} . \mathrm{H})$ and 0.644 (I. H). This is shown in Figure 1, where the variations of $\tan ^{2} \theta_{12}$ and $\tan ^{2} \theta_{23}$ are plotted against $\sin \theta_{13}$.

\section{Jarkslog Parameter $\left(J_{c p}\right)$}

We introduce the CP phase $\delta$ in $U_{13}$ as shown in Equation (8). The inclusion of $\delta_{c p}$ does not affect $\tan ^{2} \theta_{12}$ or $\tan ^{2} \theta_{23}$ (Equations (9) and (10)).

We obtain the $J_{c p}$ as,

$$
\begin{gathered}
J_{c p}=\operatorname{Im}\left[u_{e 1}^{*} u_{\mu 1}^{*} u_{e 3} u_{\mu 1}\right]=\epsilon\left(1-\epsilon^{2}\right)\left(\frac{1}{\sqrt{2}}-\frac{\epsilon}{2}\right)\left(\frac{1}{\sqrt{3}}-\frac{\epsilon}{5}\right) \\
\cdot\left(\frac{1}{2}+\frac{\epsilon}{\sqrt{2}}-\frac{\epsilon^{2}}{4}\right)^{\frac{1}{2}}\left(\frac{2}{3}+\frac{2 \epsilon}{5 \sqrt{3}}-\frac{\epsilon^{2}}{25}\right)^{\frac{1}{2}} \sin \delta
\end{gathered}
$$

Maximum $J_{c p}$, i.e., $J_{\max }$ is obtained for $\delta=\pi / 2$. For, $\epsilon=0.156, J_{\max }$ is obtained as 0.0341 . The variation of $J_{\max }$ with respect to $\sin \theta_{13}$ is shown in Figure 2. Also the variation of $J_{c p}$ with $\delta$ (with $\epsilon$ or $\sin \theta_{13}$ fixed at 0.156), is plotted in Figure 3.

\section{Generation of Neutrino Mass Matrix with Inverted Hierarchy}

We now apply the PMNS mixing matrix from Equation (7), to construct the neutrino mass matrix with inverted hierarchy (I. H). We try to interpret the masses in terms of the same parameter $\sin \theta_{13}=\epsilon$.

We choose on phenomenological ground the absolute values of three neutrino masses in units of $\mathrm{eV}$ as,

$$
\begin{gathered}
m_{1}=\frac{60}{1250}+\frac{7}{8} \epsilon^{4}, \\
m_{2}=\frac{61}{1250}+\frac{7}{8} \epsilon^{4}-\frac{\epsilon^{5}}{3}, \\
m_{3}=\frac{7}{8} \epsilon^{4} .
\end{gathered}
$$

leading to,

$$
\begin{aligned}
& \Delta m_{21}^{2}=\frac{121}{1562500}+\frac{7 \epsilon^{4}}{5000}-\frac{61}{1875} \epsilon^{5}-\frac{7}{12} \epsilon^{9}+\frac{1}{9} \epsilon^{10}, \\
& \Delta m_{23}^{2}=\frac{3721}{1562500}+\frac{427 \epsilon^{4}}{5000}-\frac{61}{1875} \epsilon^{5}-\frac{7}{12} \epsilon^{9}+\frac{1}{9} \epsilon^{10} .
\end{aligned}
$$

The variations of $\Delta m_{21}^{2}$ and $\Delta m_{23}^{2}$ with $\epsilon$ are shown in Figures 4 and 5. At TBM mixing condition, i.e., at $\epsilon=0$, we get, $\Delta m_{21}^{2}=7.74 \times 10^{-5} \mathrm{eV}^{2}$,

$\Delta m_{23}^{2}=2.38 \times 10^{-3} \mathrm{eV}^{2}$, and $\Delta m_{21}^{2}=7.53 \times 10^{-5} \mathrm{eV}^{2}$, $\Delta m_{23}^{2}=2.43 \times 10^{-3} \mathrm{eV}^{2}$ are obtained at $\epsilon=0.156$.

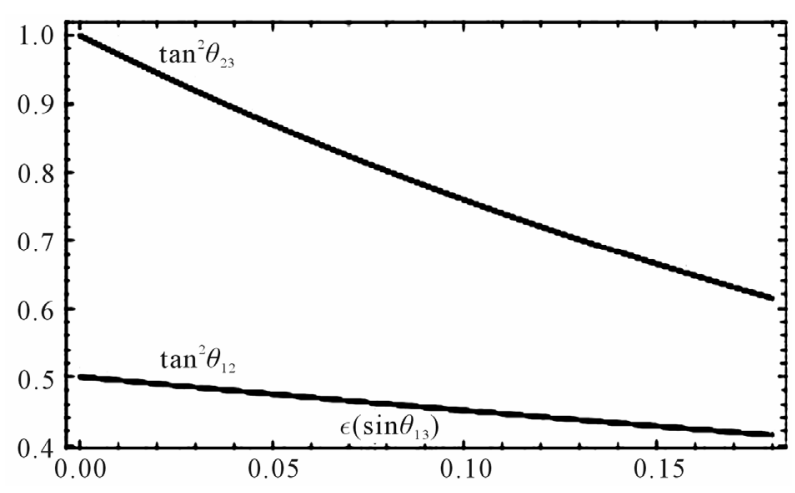

Figure 1. Variation of $\tan ^{2} \theta_{12}$ and $\tan ^{2} \theta_{23}$ with $\epsilon$.

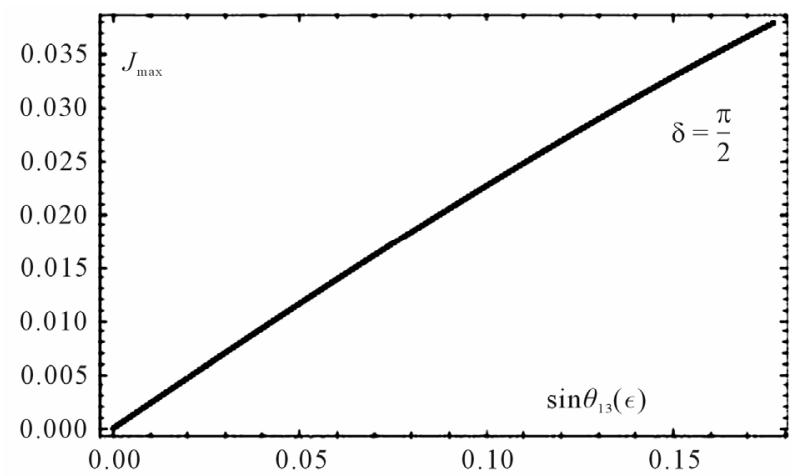

Figure 2. Variation of $J_{\max }$ with $\epsilon$.

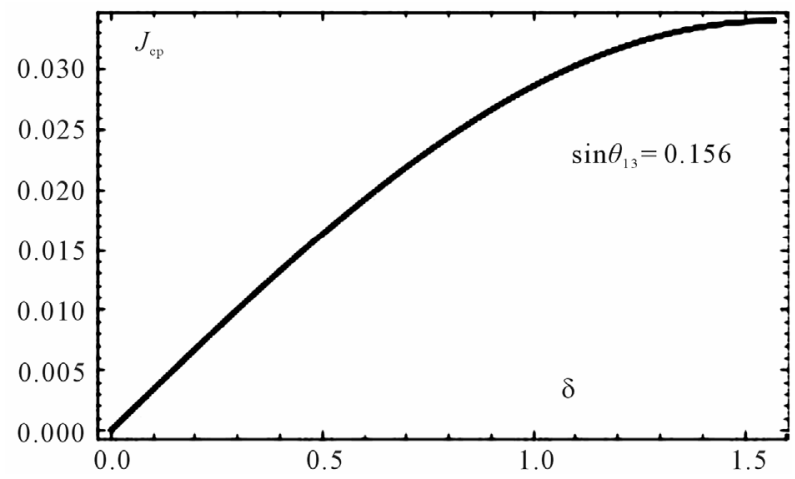

Figure 3. Variation of $J_{c p}$ with $\delta$.

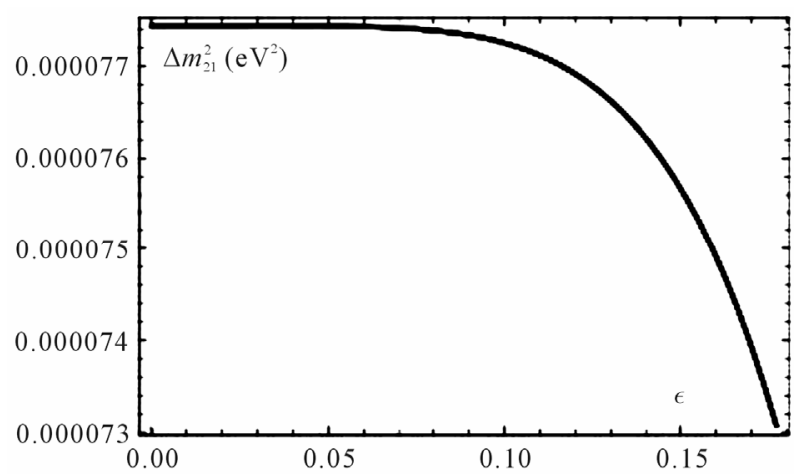

Figure 4. Variation of $\Delta m_{21}^{2}$ with $\epsilon$. 


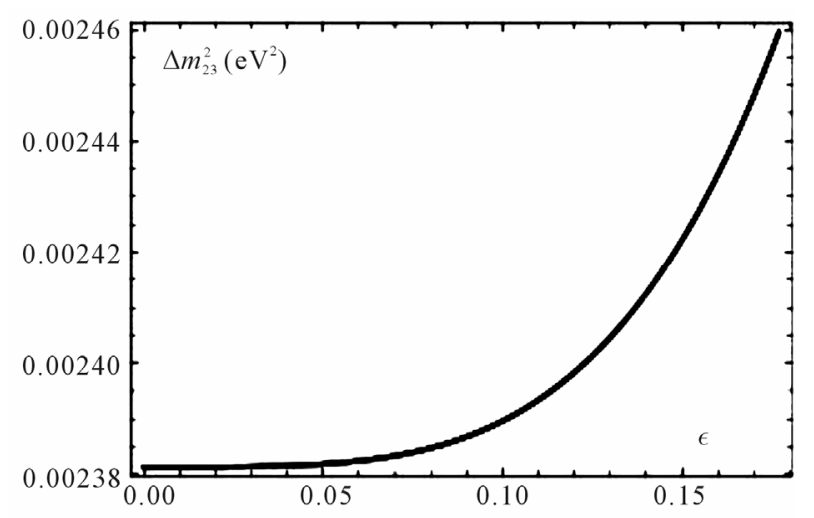

Figure 5. Variation of $\Delta m_{23}^{2}$ with $\epsilon$.
For simplicity in the texture of the neutrino mass matrix, we avoid the inclusion of $\delta_{c p}$. Using Equation (7) for $U_{\text {PMNS }}$ (with $\delta_{c p}=0$ ) and Equations (14)-(16) for $m_{i}$, we construct the neutrino mass matrix $M_{v}$ as follows,

$$
\begin{aligned}
M_{v} & =U_{\text {PMNS }}^{\mathrm{T}} \cdot\left(\begin{array}{ccc}
m_{1} & 0 & 0 \\
0 & -m_{2} & 0 \\
0 & 0 & m_{3}
\end{array}\right) \cdot U_{\text {PMNS }} \\
& =\left(\begin{array}{lll}
m_{11} & m_{12} & m_{13} \\
m_{21} & m_{22} & m_{23} \\
m_{31} & m_{32} & m_{33}
\end{array}\right) .
\end{aligned}
$$

where,

$$
\begin{aligned}
& m_{11}=\frac{7}{8} \epsilon^{6}+A^{2}(\epsilon)\left(1-\epsilon^{2}\right)\left(\frac{6}{125}+\frac{7}{8} \epsilon^{4}\right)-B(\epsilon)\left(1-\epsilon^{2}\right)\left(\frac{1}{\sqrt{3}}-\frac{\epsilon}{5}\right)^{2}, \\
& m_{12}=-\frac{7}{8} \epsilon^{5}\left(1-\epsilon^{2}\right)^{\frac{1}{2}}\left(\frac{1}{\sqrt{2}}-\frac{\epsilon}{2}\right)+A(\epsilon)\left(1-\epsilon^{2}\right)^{\frac{1}{2}}\left(\frac{6}{125}+\frac{7}{8} \epsilon^{4}\right)\left\{C(\epsilon)\left(\frac{1}{\sqrt{3}}-\frac{\epsilon}{5}\right)+A(\epsilon)\left(\frac{1}{\sqrt{2}}-\frac{\epsilon}{2}\right)\right\} \\
& +B(\epsilon)\left(1-\epsilon^{2}\right)^{\frac{1}{2}}\left(\frac{1}{\sqrt{3}}-\frac{\epsilon}{5}\right)\left\{\epsilon\left(\frac{\epsilon}{2}-\frac{1}{\sqrt{2}}\right)\left(\frac{1}{\sqrt{3}}-\frac{\epsilon}{5}\right)+C(\epsilon) A(\epsilon)\right\}, \\
& m_{13}=\frac{7}{8} C(\epsilon) \epsilon^{5}\left(1-\epsilon^{2}\right)^{\frac{1}{2}}+B(\epsilon)\left(1-\epsilon^{2}\right)^{\frac{1}{2}}\left(\frac{1}{\sqrt{3}}-\frac{\epsilon}{5}\right)\left\{\epsilon C(\epsilon)\left(\frac{1}{\sqrt{3}}-\frac{\epsilon}{5}\right)+A(\epsilon)\left(\frac{1}{\sqrt{2}}-\frac{\epsilon}{2}\right)\right\} \\
& +A(\epsilon)\left(1-\epsilon^{2}\right)^{\frac{1}{2}}\left(\frac{6}{125}+\frac{7 \epsilon^{4}}{8}\right)\left\{\left(\frac{1}{\sqrt{2}}-\frac{\epsilon}{2}\right)\left(\frac{1}{\sqrt{3}}-\frac{\epsilon}{5}\right)-\epsilon A(\epsilon) C(\epsilon)\right\} \\
& m_{22}=\frac{7}{8} \epsilon^{4}\left(1-\epsilon^{2}\right)\left(\frac{1}{\sqrt{2}}-\frac{\epsilon}{2}\right)^{2}+\left(\frac{6}{125}+\frac{7 \epsilon^{4}}{8}\right)\left\{C(\epsilon)\left(\frac{1}{\sqrt{3}}-\frac{2}{5}\right)+\epsilon A(\epsilon)\left(\frac{1}{\sqrt{2}}-\frac{\epsilon}{2}\right)\right\}^{2} \\
& -B(\epsilon)\left\{\epsilon\left(\frac{\epsilon}{2}-\frac{1}{\sqrt{2}}\right)\left(\frac{1}{\sqrt{3}}-\frac{\epsilon}{5}\right)+A(\epsilon) C(\epsilon)\right\}^{2}, \\
& m_{23}=-\frac{7 \epsilon^{4}}{8} C(\epsilon)\left(1-\epsilon^{2}\right)\left(\frac{1}{\sqrt{2}}-\frac{\epsilon}{2}\right) \\
& -B(\epsilon)\left\{\epsilon C(\epsilon)\left(\frac{1}{\sqrt{3}}-\frac{\epsilon}{5}\right)+A(\epsilon)\left(\frac{1}{\sqrt{2}}-\frac{\epsilon}{2}\right)\right\}\left\{\epsilon\left(\frac{\epsilon}{2}-\left(\frac{1}{\sqrt{3}}-\frac{\epsilon}{5}\right)+C(\epsilon) A(\epsilon)\right\}\right. \\
& +\epsilon A(\epsilon)\left(\frac{6}{125}+\frac{7 \epsilon^{4}}{8}\right)\left\{C(\epsilon)\left(\frac{1}{\sqrt{3}}-\frac{\epsilon}{5}\right)+\left(\frac{1}{\sqrt{2}}-\frac{\epsilon}{2}\right)\right\}\left\{\left(\frac{1}{\sqrt{2}}-\frac{\epsilon}{2}\right)\left(\frac{1}{\sqrt{3}}-\frac{\epsilon}{5}\right)-\epsilon C(\epsilon) A(\epsilon)\right\}, \\
& m_{33}=-\frac{7 \epsilon^{4}}{8} C(\epsilon)\left(1-\epsilon^{2}\right)-B(\epsilon)\left\{\epsilon C(\epsilon)\left(\frac{1}{\sqrt{3}}-\frac{\epsilon}{5}\right)+A(\epsilon)\left(\frac{1}{\sqrt{2}}-\frac{\epsilon}{2}\right)\right\}^{2} \\
& +\left(\frac{6}{125}+7 \frac{\epsilon^{4}}{8}\right)\left\{\left(\frac{1}{\sqrt{2}}-\frac{\epsilon}{2}\right)\left(\frac{1}{\sqrt{3}}-\frac{\epsilon}{5}\right)-\epsilon C(\epsilon) A(\epsilon)\right\}^{2} .
\end{aligned}
$$


and,

$$
\begin{gathered}
A(\epsilon)=\left(\frac{2}{3}+\frac{2 \epsilon}{5 \sqrt{3}}-\frac{\epsilon^{2}}{25}\right)^{\frac{1}{2}}, \\
B(\epsilon)=\left(\frac{61}{1250}+\frac{7 \epsilon^{4}}{8}-\frac{\epsilon^{5}}{3}\right)^{\frac{1}{2}}, \\
C(\epsilon)=\left(\frac{1}{2}+\frac{\epsilon}{\sqrt{2}}-\frac{\epsilon^{2}}{4}\right)^{\frac{1}{2}} .
\end{gathered}
$$

At, $\epsilon=0$ (T. B. M mixing), Equation (22) reduces to $\mu-\tau$ symmetric mass matrix form,

$$
M_{\mu \tau}=\left(\begin{array}{ccc}
\delta_{1} & 1 & 1 \\
1 & \delta_{2} & \delta_{2} \\
1 & \delta_{2} & \delta_{2}
\end{array}\right) m_{0},
$$

with $\delta_{1,2} \ll 1$, for inverted hierarchy. Equation (19) leads to

$$
\begin{gathered}
M_{v}=M_{\mu \tau}=\frac{121}{3750}\left(\begin{array}{ccc}
\frac{1}{2} & 1 & 1 \\
1 & -\frac{1}{4} & -\frac{1}{4} \\
1 & -\frac{1}{4} & -\frac{1}{4}
\end{array}\right) \\
=\left(\begin{array}{lll}
0.0157 & 0.0323 & 0.0323 \\
0.0323 & -0.0083 & -0.0083 \\
0.0323 & -0.0083 & -0.0083
\end{array}\right), \\
m_{1}=\frac{60}{1250}, m_{2}=-\frac{61}{1250}, m_{3}=0 \mathrm{in} \mathrm{eV}
\end{gathered}
$$

At $\epsilon=0.156$, Equation (22) leads to

$$
\begin{aligned}
M_{v} & =M_{\mu \tau}+\Delta M_{v} \\
& =\left(\begin{array}{ccc}
0.0189 & 0.0362 & 0.0255 \\
0.0362 & -0.0049 & -0.0118 \\
0.0255 & -0.0118 & -0.0142
\end{array}\right) .
\end{aligned}
$$

where,

$$
\begin{gathered}
\Delta M_{v}=\left(\begin{array}{ccc}
0.0032 & 0.0039 & -0.0068 \\
0.0039 & 0.0034 & -0.0035 \\
-0.0068 & -0.0035 & -0.0059
\end{array}\right) \\
m_{1}=0.0485, m_{2}=-0.0493, m_{3}=0.0005 \text { in eV. }
\end{gathered}
$$

\section{Summary}

We have started with a parameter $\epsilon$ equating this to $\sin \theta_{13}$ and construct the PMNS matrix, $U_{\text {PMNS }}$. Then we represent the neutrino masses $\left(m_{i=1,2,3}\right)$ in terms of the same parameter $\sin \theta_{13}$, i.e. $\epsilon$. We verify our hypo- thesis by comparing the ranges of the mass squared differences as a result of our ansatz with the $1 \sigma$ range, experimentally obtained. We take the range of $\epsilon$ as the experimental $1 \sigma$ range of $\sin \theta_{13}$ [6]. We obtain the range of $\Delta m_{21}^{2}$ and $\Delta m_{23}^{2}$ as $(7.46-7.58) \times 10^{-5} \mathrm{eV}^{2}$ and $(2.42-2.44) \times 10^{-3} \mathrm{eV}^{2}$ respectively. The respective ranges obtained, lie within the experimental $1 \sigma$ boundary [6]. This provides a support to our hypothesis $m_{i}$ as $m_{i}(\epsilon)$. This is to be emphasised that the $U_{\text {PMNS }}$ matrix as proposed in Equation (7) satisfies the unitary condition and is not dependent on the choice of the order of $\epsilon$. The introduction of $\delta_{c p}$ does not affect $\tan ^{2} \theta_{12}$ and $\tan ^{2} \theta_{23}$ in our calculation. The maximum $J_{c p}$ obtained is 0.034 (with respect to $\epsilon=\sin \theta_{13}=0.156$ ). Finally we concentrate on the construction of $M_{v}$, the neutrino mass matrix. The present investigation though phenomenological, gives a complete picture of the texture of the neutrino mass matrix which can be employed in other applications regarding baryon asymmetry of the universe [18]. Although we have constructed the mass matrix for inverted hierarchical model, yet we can extend our technique to Normal as well as Quasidegenerate mass models.

\section{REFERENCES}

[1] Y. Abe, et al., "Indication for the Disappearance of Reactor Electron Antineutrinos in the Double Chooz Experiment," Physical Review Letters, Vol. 108, No. 13, 2012, pp. 131801-131807.

doi:10.1103/PhysRevLett.108.131801

[2] F. An, et al., "Observation of Electron-Antineutrino Disappearance at Daya Bay," Physical Review Letters, Vol. 108, No. 17, 2012, pp. 171803-171809.

[3] J. Ahn, et al., "Observation of Reactor Electron Antineutrino Disappearance in the RENO Experiment," Physical Review Letters, Vol. 108, No. 18, 2012, Article ID: 191802. doi:10.1103/PhysRevLett.108.191802

[4] K. Abe, et al., "Indication of Electron Neutrino Appearance from an Accelerator Produced Off-Axis Muon Neutrino Beam," Physical Review Letters, Vol. 107, No. 4, 2011, Article ID: 041801. doi:10.1103/PhysRevLett.107.041801

[5] R. Nichol, "Plenary Talk at the Neutrino 2012 Conference," 2012. http://neu2012.kek.jp/

[6] G. L. Fogli, E. Lisi, A. Marrone, D. Montanino, A. Palazzo and A. M. Rotunno, "Global Analysis of Neutrino Masses, Mixings and Phases: Entering the Era of Leptonic CP Violation Searches," Physical Review D, Vol. 86, No. 1, 2012, Article ID: 013012. doi:10.1103/PhysRevD.86.013012

[7] P. F. Harrison, D. H. Perkins and W. G. Scott, "Tri-Bimaximal Mixing and the Neutrino Oscillation Data," Physics Letters B, Vol. 530, No. 1-4, 2002, pp. 167-173. doi:10.1016/S0370-2693(02)01336-9

[8] K. S. Babu, E. Ma and J. W. F. Valle, "Underlying $A_{4}$ 
Symmetry for the Neutrino Mass Matrix and the Quark Mixing Matrix," Physics Letters B, Vol. 552, No. 3-4, 2003, pp. 207-213. doi:10.1016/S0370-2693(02)03153-2

[9] E. Ma, "The All-Purpose Neutrino Mass Matrix," Physical Review D, Vol. 66, No. 11, 2002, pp. 11730111117303. doi:10.1103/PhysRevD.66.117301

[10] G. Altarelli and F. Feruglio, "Tri-Bimaximal Neutrino Mixing from Discrete Symmetry in Extra Dimensions," Nuclear Physics B, Vol. 720, No. 1-2, 2005, pp. 64-88. doi:10.1016/j.nuclphysb.2005.05.005

[11] G. Altarelli and F. feruglio, "Tri-Bimaximal Neutrino Mixing, $\mathrm{A}_{4}$ and the Modular Symmetry," Nuclear Physics $B$, Vol. 741, No. 1-2, 2006, pp. 215-235. doi:10.1016/j.nuclphysb.2006.02.015

[12] G. Altarelli and F. feruglio, "Phenomenology of Neutrino Masses and Mixings," Acta Physica Polonica B, Vol. 30, 1999, pp. 3067-3088.

[13] E. Ma and D. Wegman, "Nonzero $\theta_{13}$ for Neutrino Mixing in the Context of $\mathrm{A}_{4}$ Symmetry," Physical Review Letters, Vol. 107, No. 6, 2011, Article ID: 061803. doi:10.1103/PhysRevLett.107.061803
[14] H. Ishimori and E. Ma, "New Simple $A_{4}$ Neutrino Model for Nonzero $\theta_{13}$ and Large $\delta_{C P}$," Physical Review D, Vol. 86, No. 4, 2012, Article ID: 045030. doi:10.1103/PhysRevD.86.045030

[15] S. M. Boucenna, S. Morisi, M. Tortola and J. W. F. Valle, "Bi-Large Neutrino Mixing and the Cabibbo Angle," Physical Review D, Vol. 86, No. 5, 2012, Article ID: 051301. doi:10.1103/PhysRevD.86.051301

[16] E. Ma, A. Natale and A. Rashed, "Scotogenic $A_{4}$ Neutrino Model for Nonzero $\theta_{13}$ and Large $\delta_{C P}$," International Journal of Modern Physics A, Vol. 27, No. 23, 2012, Article ID: 1250134. doi:10.1142/S0217751X12501345

[17] D. Marzocca, S. T. Petcov, A. Romanino and M. Spinrath, "Sizeable $\theta_{13}$ from the Charged Lepton Sector in SU(5), (Tri-)Bimaximal Neutrino Mixing and Dirac CP Violation," Journal of High Energy Physics, Vol. 2011, No. 9, 2011, in press. doi:10.1007/JHEP11(2011)009

[18] N. K. Francis and N. N. Singh, "Validity of Quasi-Degenerate Neutrino Mass Models and Their Predictions on Baryogenesis," Nuclear Physics B, Vol. 863, No. 1, 2012, pp. 19-32. doi:10.1016/j.nuclphysb.2012.05.017 